\title{
Design of Electro-optic Mach-zehnder Interferometer Based All-optical Binary Half Adder and Half Subtractor
}

sehajpal kaur ( $\nabla$ sehajpal.ece@gndu.ac.in )

GNDU: Guru Nanak Dev University

Maninder Lal Singh

Guru nanak Dev university

Priyanka .

Guru Nanak Dev University

Mandeep Singh

Guru Nanak Dev University

\section{Research Article}

Keywords: Switching phenomena, half-adder and half-subtractor, Beam propagation method, MachZehnder interferometer

Posted Date: April 20th, 2021

DOl: https://doi.org/10.21203/rs.3.rs-404604/v1

License: (c) (i) This work is licensed under a Creative Commons Attribution 4.0 International License. Read Full License 


\title{
Design of Electro-optic Mach-Zehnder Interferometer based All-Optical Binary Half Adder and Half Subtractor
}

\author{
Sehajpal Kaur ${ }^{1}$, Maninder Lal Singh ${ }^{2}$, Priyanka ${ }^{3}$, Mandeep Singh ${ }^{4}$ \\ Department of Electronics Technology \\ Guru Nanak Dev University, Amritsar, India \\ ${ }^{1}$ sehajpal.ece@gndu.ac.in, ${ }^{2}$ mlsingh.ece@gndu.ac.in, ${ }^{3}$ priyankaece.rsh@gndu.ac.in, ${ }^{4}$ mandeepece.rsh@gndu.ac.in
}

\begin{abstract}
By exploiting the phenomena of optical switching, different logic functions for all-optical digital signal processing has been projected. This paper presents the application of optical switching to design of all-optical half adder and half subtractor by Mach-Zehnder interferometer. All-Optical half adder and half subtractor are designed with the optimized structure of $2 \times 2$ Mach-Zehnder interferometer switch by electro-optic effect in lithium niobate. Numerical simulations of the proposed structure have been conducted to verify the suitability of the designed structure using Opti-BPM software. The implementation of proposed structures is simulated in MATLAB software along with the mathematical description. It is interesting to analyze that the proposed structures are useful to generate combinational and sequential logic circuits in high-speed optical signal processing and switching network.
\end{abstract}

Keywords: Switching phenomena, half-adder and half-subtractor, Beam propagation method, Mach-Zehnder interferometer.

\section{Declarations}

Funding (information that explains whether and by whom the research was supported) Conflicts of interest/Competing interests (No)

Availability of data and material (Yes)

Code availability (Yes)

Authors' contributions (No)

1. Introduction

The expansion of optical signal processing technology is necessary for future high-speed optical systems for signal manipulation to implement all-optical signal processing functions includes switching, multiplexing/demultiplexing, coding/decoding, logic gates, add/drop, multicasting etc. without optical to electronic conversion [1]. The rapid growth in switching technology, enhance the energy requirement and speed of operation in optical communication system [2]. All-optical switching in non-linear optics can be easily employed by an optical waveguide to maintain high-intensity light over a long distance which is limited in free space optics due to diffraction. Switching technology can be classified based on physical effect, device design and material used such as Electro-Optic, Acousto-Optic, Thermo-Optic, Optomechanical and Optical Amplifier based switching. Out of these different switching technologies, electro-optic switching is employed in optical waveguides and directional couplers using popular electro-optic material Lithium-niobate (high electro-optic co-efficient) and Gallium Arsenide (low electro-optic co-efficient) having low attenuations and low power consumption in optical communication wavelengths. They are capable of fast switching speed in the range of microseconds to tens of nanoseconds by electro-optic effect [3-4]. MachZehnder interferometer (MZI) and multimode interferometer (MMI) are the most popular 
optical waveguide device used to implement all-optical digital processing circuits by an electro-optic effect. By using the phenomena of electro-optic effect in lithium niobite substrate the refractive index of a material is modulated by applying an external electric field to the interferometric arms. In recent years researchers focus to implement all-optical logic and arithmetic operations with mach-zehnder interferometer switch in optical signal processing. All-optical XOR/XNOR and AND gate has bee jn reported using Mach-Zehnder interferometer by electro-optic effect[5]. The universal logic gates have been investigated in terms of speed, latency and power consumption using a cascaded Mach-Zehnder interferometer switch structure [6]. By phase encoding using four wave mixing (FWM) in semiconductor optical amplifier (SOA), half addition and half subtraction are performed to implement superfast arithmetic logic unit (ALU) [7]. All-optical full adder with ER and Q-factor of $11.7 \mathrm{~dB}$ and 12.5, is implemented using QD-SOA based mach-zehnder interferometer as a basic building block to perform other signal processing function [8]. Phase encoding scheme has been exploited to perform half addition and half subtraction to implemented digital comparator [9]. SOA-based all-optical half adder has experimentally demonstrated at $10 \mathrm{Gbit} / \mathrm{s}$ [10]. Alloptical half adder structure is proposed in a two-dimensional photonic crystal structure using non-linear resonant cavities [11]. Similarly, non-linear photonic crystal-based ring resonator is used to implement half subtractor [12].

In this communication, Mach-Zehnder interferometer-based electro-optic switching configurations have been used to design half adder and half subtractor. The aim of this paper to realize half adder and half subtractor using a minimum number of Mach Zehnder interferometer switching configurations. The article is organized as follows: Section 2, describes the basic concept and operation principle of mach-zehnder interferometer. Section 3, is concerned with layout design structure and simulation results of the proposed half adder and half subtractor with the help of Beam propagation method (BPM) and MATLAB. Finally, the conclusion is drawn in sect 4.

\section{Design Concept and operational principle of Mach-Zehnder interferometer}

Many other different approaches have been proposed to design all-optical digital logic functions based on Periodically Poled Lithium Niobate Waveguide (PPLN) [13, 14], microring and nanoring devices [15], a terahertz optical asymmetric demultiplexer (TOAD) [16], non-linear directional coupler [17], non-linear effects in SOA-MZI[18-25]. Among these different technologies, Mach-Zehnder interferometer electro-optic switching configurations represents the best solution to implement all-optical logic functions using a conversion of phase modulation into intensity modulation because of compact size, low power consumption, low latency and thermal stability. Mach-Zehnder interferometer switch consists of one $2 \times 2$ optical splitter at the input ports and one $2 \times 2$ optical combiner at the output ports. Two interferometer arms are connected between splitter and combiner as depicted in figure 1. An optical signal is injected into input ports, which divide equally over the interferometer arms by splitter. The signal divides equally at the output of $2 \times 2$ splitter has a phase difference of $\pi / 2 \mathrm{rad}$. A constructive and destructive interference at the cross output port and bar output port are introduced if the two interferometer arms are equal. In order to switch the signal from one output port to another, a phase shift $\pi$ is required to introduce in one of the interferometer arms. For phase modulation to occur, these switches use metal electrodes over the interferometric arms to create refractive index variations by applying an electric field to the electrodes. One of the physical phenomena that can provide the required phase shift is electro-optic effect [26]. By utilizing EO-effect, mach-zehnder interferometer exhibits stable performance parameters 
for a wide range of optical power. Figure 1 shows the schematic layout of Mach-Zehnder interferometer-based electro-optic switch.

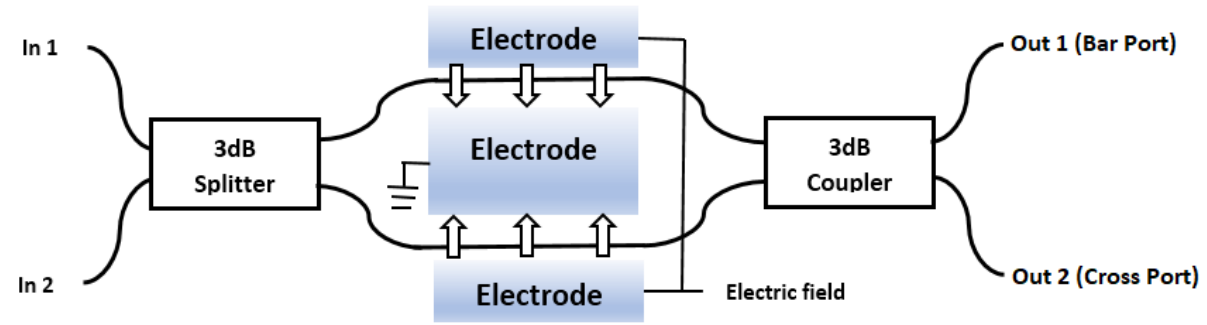

Fig. 1. Schematic view of electro-optic MZI switch

As shown in figure 1, mach-zehnder interferometer switch is made by $\mathrm{LiNbO}_{3}$ optical waveguide and a pair of electrodes. The phase change induced by electro-optic effect having electrode length $\mathrm{L}$, a separation between two electrodes d due to applied voltage $\mathrm{V}$ is given as

$$
\Delta \varphi=\frac{2 \Pi}{\lambda}\left[\left(\frac{n^{3}}{2}\right) \frac{V}{d} r\right]
$$

The amount of normalized power at different output ports of Mach-Zehnder interferometer can be obtained by electro-optic effect as follows:

$$
\begin{aligned}
& P_{\text {OUT1 }}=\left|j e^{-j\left(\varphi_{0}\right)} \sin \frac{\Delta \varphi}{2}\right|^{2}=\sin ^{2}\left(\frac{\Delta \varphi}{2}\right) \\
& P_{\text {OUT2 }}=\left|j e^{-j\left(\varphi_{0}\right)} \cos \frac{\Delta \varphi}{2}\right|^{2}=\cos ^{2}\left(\frac{\Delta \varphi}{2}\right)
\end{aligned}
$$

3. Description and mathematical formulation of half adder and half subtractor using OptiBPM

A half adder and half subtractor are combinational logic circuits that can perform addition and subtractions of two binary digits. A half adder gives two outputs Sum and Carry, similarly a half subtractor generates two outputs Difference and Borrow. To develop half adder and half subtractor, a basic Mach-Zehnder interferometer based electro-optic switch has been designed at a wavelength of $1.46 \mu \mathrm{m}$ in Opti-BPM software [27]. Opti-BPM is based on FD-BPM that provides a complete analysis of optical waveguide devices and optical fiber including refractive index distribution, waveguide material, coupling, splitting, multiplexing and demultiplexing of optical signals [28].

\subsection{Design of Half adder using electro-optic effect based MZI structure}

Fig. 2 shows the Opti-BPM layout structure of the half adder. As shown in fig. 2, a half adder adds two binary numbers (A and B) and gives two binary outputs called $\operatorname{Sum}(\mathrm{S})$ and $\operatorname{Carry}(\mathrm{C})$. The device has three MZIs with two control signals $\mathrm{X}$ and $\mathrm{Y}$. The control signals $\mathrm{X}$ and $\mathrm{Y}$ are the two electrode voltages applied at the center electrode of MZIs. Using different combinations of control signals $\mathrm{X}$ and $\mathrm{Y}$ that are used to represent half adder the output power is obtained at different output ports. 


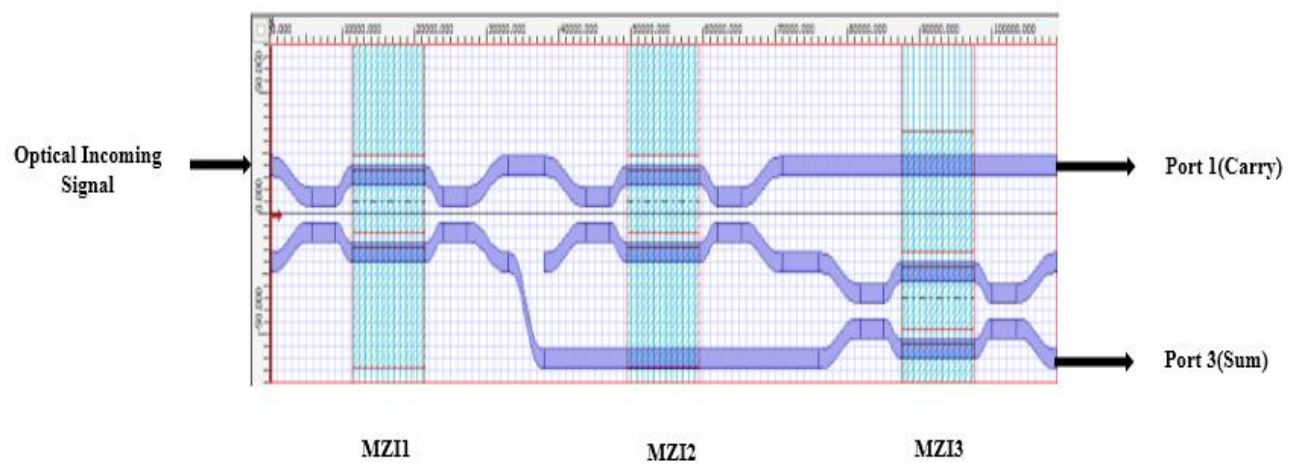

Fig. 2. Opti-BPM layout of Half Adder using MZI switch

\subsubsection{Normalized output power at various output ports of half adder}

Using the output power relationship of the single-stage Mach-Zehnder interferometer in equations 2 and 3, the normalized output power at output port 3 and port 1 can be represented by combining all possible minterms shown in table 1 .

For Sum:

$$
\begin{aligned}
\text { Output port } 3=m_{1}+m_{2}=\left|\frac{O U T 2_{M Z I 3}}{E_{\text {in }}}\right|^{2}= & \cos ^{2}\left(\frac{\Delta \varphi_{M Z I 1}}{2}\right) \times \sin ^{2}\left(\frac{\Delta \varphi_{M Z I 3}}{2}\right)+ \\
& \sin ^{2}\left(\frac{\Delta \varphi_{M Z I 1}}{2}\right) \times \cos ^{2}\left(\frac{\Delta \varphi_{M Z I 2}}{2}\right)
\end{aligned}
$$

For Carry:

Output port $1=m_{3}=\left|\frac{O U T 1_{M Z I 2}}{E_{\text {in }}}\right|^{2}=\sin ^{2}\left(\frac{\Delta \varphi_{M Z I 1}}{2}\right) \times \sin ^{2}\left(\frac{\Delta \varphi_{M Z I 2}}{2}\right)$

Table 1 Truth table of half-adder for different combinations of control signals.

\begin{tabular}{|c|c|c|c|}
\hline $\begin{array}{c}\text { Control signal X } \\
\text { Input A }\end{array}$ & $\begin{array}{c}\text { Control signal Y } \\
\text { Input B }\end{array}$ & Port 3 (Sum) & Port 1 (Carry) \\
\hline 0 & 0 & 0 & 0 \\
\hline 0 & 1 & 1 & 0 \\
\hline 1 & 0 & 1 & 0 \\
\hline 1 & 1 & 0 & 1 \\
\hline
\end{tabular}

Various combination of control signals $\mathrm{X}$ and $\mathrm{Y}$ and their corresponding response applied to the given structure are used to represent half adder are discussed as follow

Case 1: $\mathrm{X}=0, \mathrm{Y}=0$

Here, the optical input signal is incident on the first input port of MZI1. The control signal $\mathrm{X}$ applied to the second electrode of MZI1 is low $(0 \mathrm{~V})$, so optical power emerges out at output port 2 of MZI1. The output power emerges out at output port 2 of MZI1 represents the input to MZI3. Now, if the control signal Y applied to MZI3 is also low, then the signal emerges out at output port 1 of MZI3. Hence, no optical signal appears at output port 1 and output port 3 of the structure shown in figure 2 represented by table 1 .

Case 2: $\mathrm{X}=0, \mathrm{Y}=1$

In this case, a continuous optical input signal is provided at input port 1 of MZI1. As a low control signal is provided at the second electrode of MZI1 (i.e X=0), allows optical signal 
appears at the output port 2 of MZI1 and optical power comes out at port 1 of MZI1 becomes 0 . The output signal appears at output port 2 of MZI1 again becomes the input of MZI3. If the control signal applied to the second electrode of MZI3 is high (Y=1), the optical signal appears at output port 3 of MZI3 represents the sum of two bits. Hence, for $\mathrm{X}=0$ and $\mathrm{Y}=1$, the output power at output port $1=0$ and output port $3=1$ of MZI3.

Case 3: $\mathrm{X}=1, \mathrm{Y}=0$

In this case, the control signal applied to the second electrode of MZI1 is high $(X=1)$, the optical signal again appears at the output port 1 of MZI1 that acts at the input of MZI2. Since control signal $\mathrm{Y}=0$ at MZI2, the output signal gets out at output port 2 of MZI2.

Now, the output signal at port 2 of MZI2 becomes the input of MZI3. For control signal $\mathrm{Y}=0$ applied to MZI3, the optical signal appears at output port 2 of MZI3 represents again the sum of two bits.

Case 4: $\mathrm{X}=1, \mathrm{Y}=1$

In a similar manner, the combination of control signals $X=1$ and $Y=1$, the optical signal observed at the output port 1 of MZI. The signal appears at output port1 of MZI1 becomes the input of MZI2. As the control signal Y applied to the center electrode of MZI2 is high, the output power appears at output port 2 of MZI2. Therefore, due to the presence of each control signal the optical power appears at output port 1 of MZI2 represents carry $=1$ and $\operatorname{sum}=0$.

Table 1 represents the truth table of all possible combinations of the control signal and output optical signal obtained at the output port 1 and port 3 of fig. 2 . It has been shown that to obtain the SUM and Carry, minterms can be combined at output port3 and output port 1. The Opti-BPM simulation results of the proposed device can be represented in table 2 . Similarly, the MATLAB simulation results obtained are represented in fig. 3. From fig. 3 the First and second row represents the all possible combination of control signals $X$ and Y provided to MZI1, MZI2 and MZI3. The third and fourth row of fig. 4 represents the SUM and CARRY of the half adder for given combinations of control signals. From OptiBPM results shown in table 2 and MATLAB results in fig. 3, it has been shown that the results obtained from Opti-BPM are exactly matched with the simulation results obtained in MATLAB for the proposed structure.

Table 2 Simulation results for half adder for different control signals using Opti-BPM

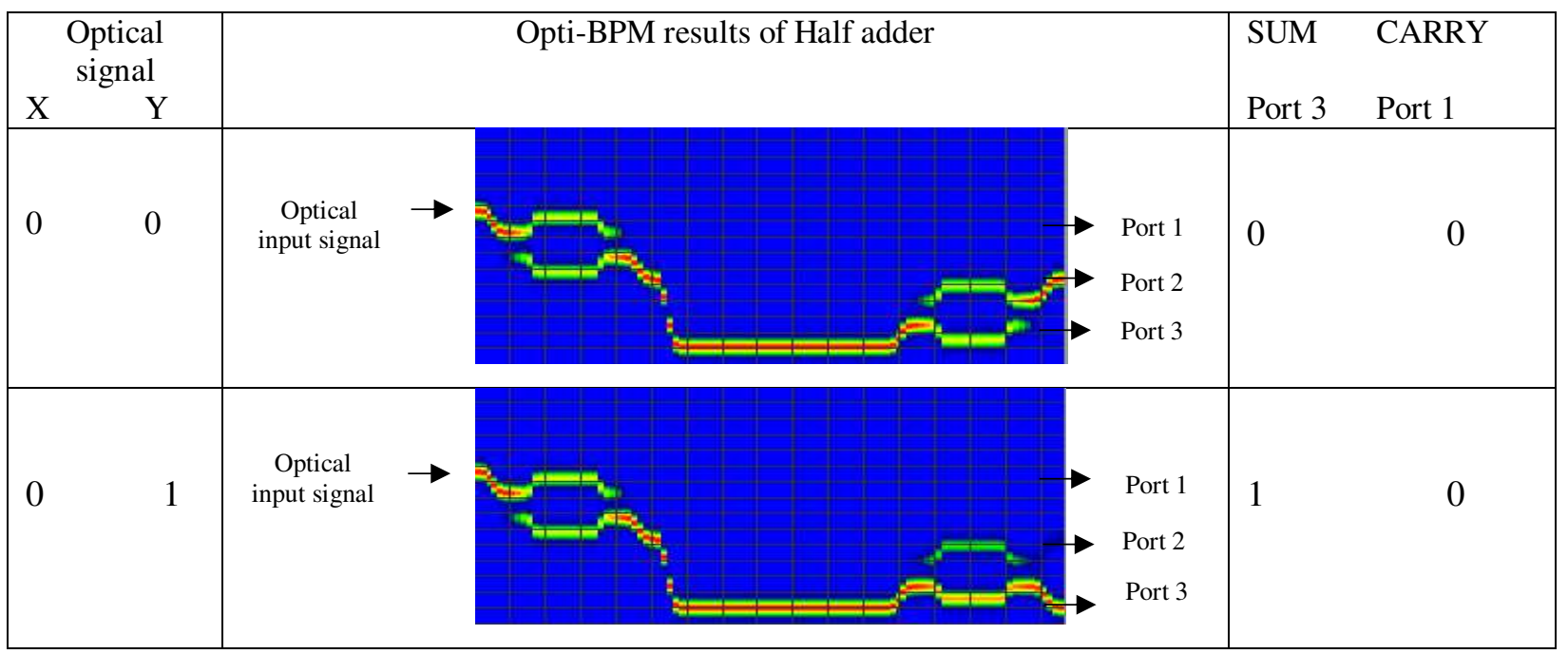




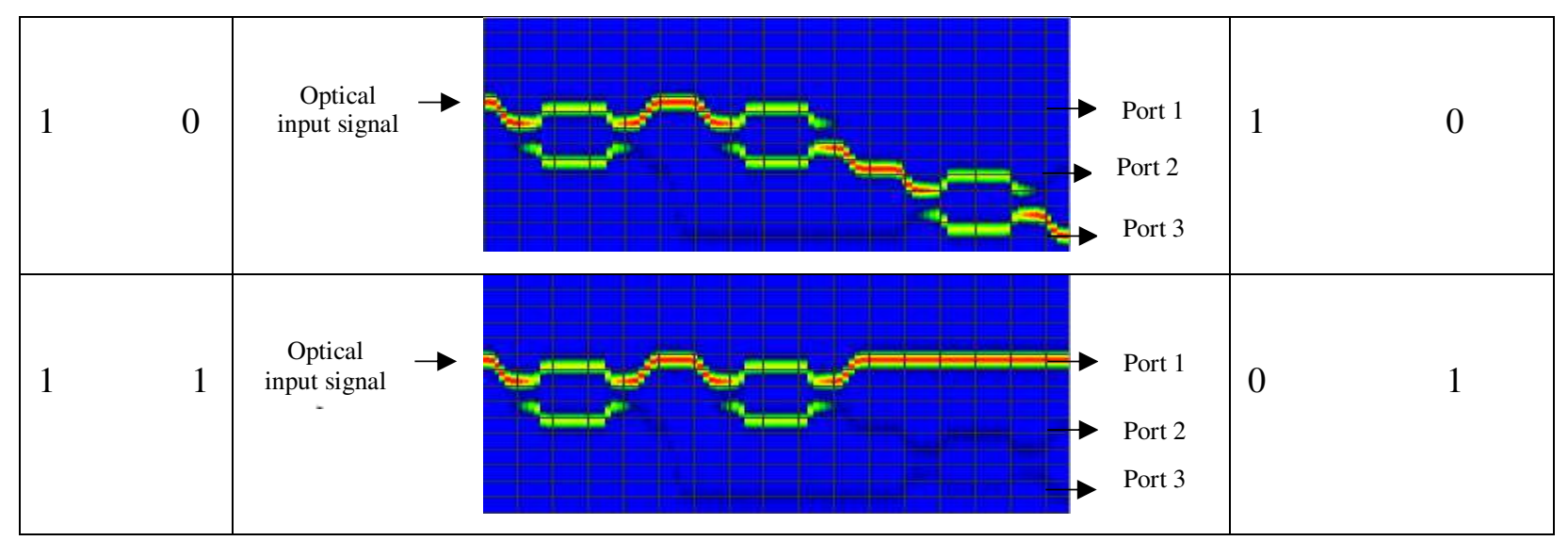
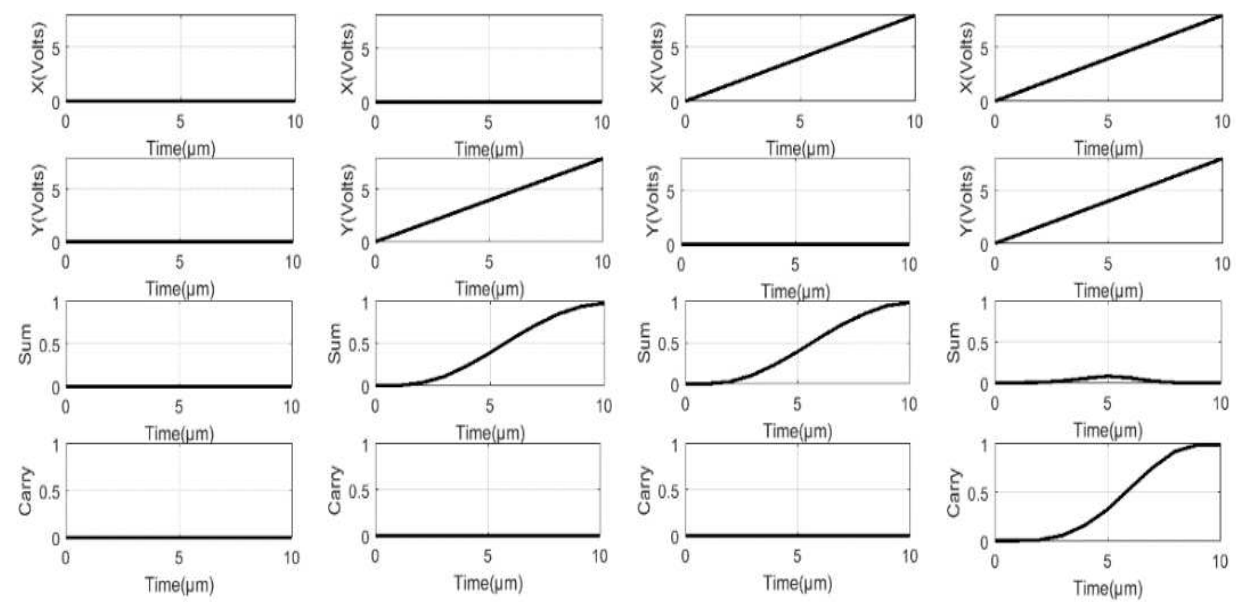

Fig. 3. MATLAB simulation results of Half Adder.

\subsection{Design of Half subtractor using electro-optic effect based MZI structure}

Figure 4 represents the design of a 2-bit half subtractor that performs subtraction of two singlebit numbers using Mach-Zehnder interferometer. The proposed design consists of three MZIs with two control signals $\mathrm{X}$ and $\mathrm{Y}$ as inputs and produce outputs as difference and borrow.

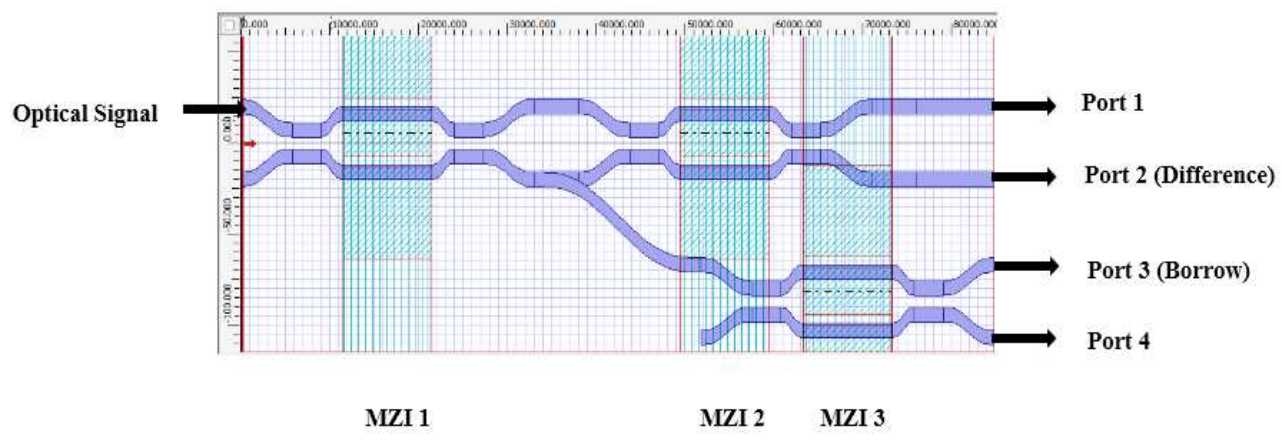

Fig. 4. Layout of Half Subtractor

\subsubsection{Normalized output power at various output ports of half subtractor}

To perform half subtraction, the output expression of normalized output power at port 2 and port 3 can be calculated using the expression of single-stage MZI: 
For Difference:

$$
\begin{array}{r}
\text { Output port 2= } m_{1}+m_{2}=\mid \frac{\text { OUT1 }\left.1_{M Z I I_{1}}\right|^{2}=}{E_{\text {in }}} \cos ^{2}\left(\frac{\Delta \varphi_{M Z I 1}}{2}\right) \times \sin ^{2}\left(\frac{\Delta \varphi_{M Z I 2}}{2}\right)+ \\
\sin ^{2}\left(\frac{\Delta \varphi_{M Z I 1}}{2}\right) \times \cos ^{2}\left(\frac{\Delta \varphi_{M Z I 2}}{2}\right)
\end{array}
$$

For Borrow:

$$
\text { Output port } 3=m_{1}=\left|\frac{O U T 1_{M Z I 3}}{E_{\text {in }}}\right|^{2}=\operatorname{Cos}^{2}\left(\frac{\Delta \varphi_{M Z I 1}}{2}\right) \times \sin ^{2}\left(\frac{\Delta \varphi_{M Z I 3}}{2}\right)
$$

The proposed design of the half subtractor consists of three Mach-zehnder interferometer is shown in fig. 4 . The optical signal is given to the first input port of MZI1. The output ports of MZI1 are further connected to MZI2 and MZI3. Binary inputs are the control signals X and Y are in the form of electric signals to MZI1, MZI2 and MZI3. The output obtained for different combinations of control signal are discussed below:

Case 1: $\mathrm{X}=0, \mathrm{Y}=0$

Figure 4 represents the optical signal is incident on input port 1 of MZI1. As the control signal, $\mathrm{X}$ provided to MZI1 is low, the optical signal appears at output port 2 of MZI1. This port is further connected to input port 2 and input port 1 of MZI2 and MZI3. Since the control signal Y provided to MZI2 and MZI3 is also low, the output appears at output port 1 and output port 2 of MZI2 and MZI3.

Case $2: \mathrm{X}=0, \mathrm{Y}=1$

In this case, the control signal X provided to MZI1 is low again. Due to this, the optical signal again appears at output port 2 of MZI1. As the voltage applied to the center electrode of MZI2 and MZI3 is high ( $Y=1)$, the output signal appears at output port 2 and output port 1 of MZI2 and MZI3 represents difference and borrow.

Case $3: \mathrm{X}=1, \mathrm{Y}=0$

When high voltage control signal $\mathrm{X}$ is provided to MZI1, the output signal emerging from output port 1 of MZI1. This output signal is the input provided to input port 1 of MZI2. Finally, due to the absence of control signal $\mathrm{Y}(\mathrm{Y}=0)$ provided to $\mathrm{MZI} 2$, the output signal comes out at output port 2 of MZI2 represents the difference of 2 bits.

Case 4: $\mathrm{X}=1$ and $\mathrm{Y}=1$

Similarly, in this case, the combination of control signals provided to MZI1, MZI2 and MZI3 is high. The light signal appears at the output port 1 of MZI1. The optical signal that appears at output port 1 of MZI1 will go to input port 1 of MZI2. Due to the presence of control signal $Y=1$, the output signal will go straight and appears at output port 1 of MZI2.

Table 3 Different combinations of control signals for half subtractor

\begin{tabular}{|c|c|c|c|}
\hline $\begin{array}{c}\text { Control signal } \\
\mathrm{X}\end{array}$ & $\begin{array}{c}\text { Control signal } \\
\mathrm{Y}\end{array}$ & $\begin{array}{c}\text { Port2 } \\
\text { (Difference) }\end{array}$ & $\begin{array}{c}\text { Port 3 } \\
\text { (Borrow) }\end{array}$ \\
\hline 0 & 0 & 0 & 0 \\
\hline 0 & 1 & 1 & 1 \\
\hline 1 & 0 & 1 & 0 \\
\hline 1 & 1 & 0 & 0 \\
\hline
\end{tabular}


Table 3 shows different combinations of control signal $\mathrm{X}$ and $\mathrm{Y}$ provided to the proposed design and their corresponding results at port 2 (difference) and port 3 (Borrow). Table 4 shows Opti-BPM results for half Subtractor obtained by providing different combinations of control signals. In similar manner, MATLAB simulation results are represented in figure 5. From the figure 5, it has been seen that first and second row represents control signal $X$ and $Y$ provided to MZIs, and the last two rows represent the difference and borrow outputs. It is apparent from the figure that MATLAB simulation results are exactly matched with the Opti-BPM results obtained in table 4.

Table 4 Results for half-subtractor for different control signals obtained through Opti-BPM

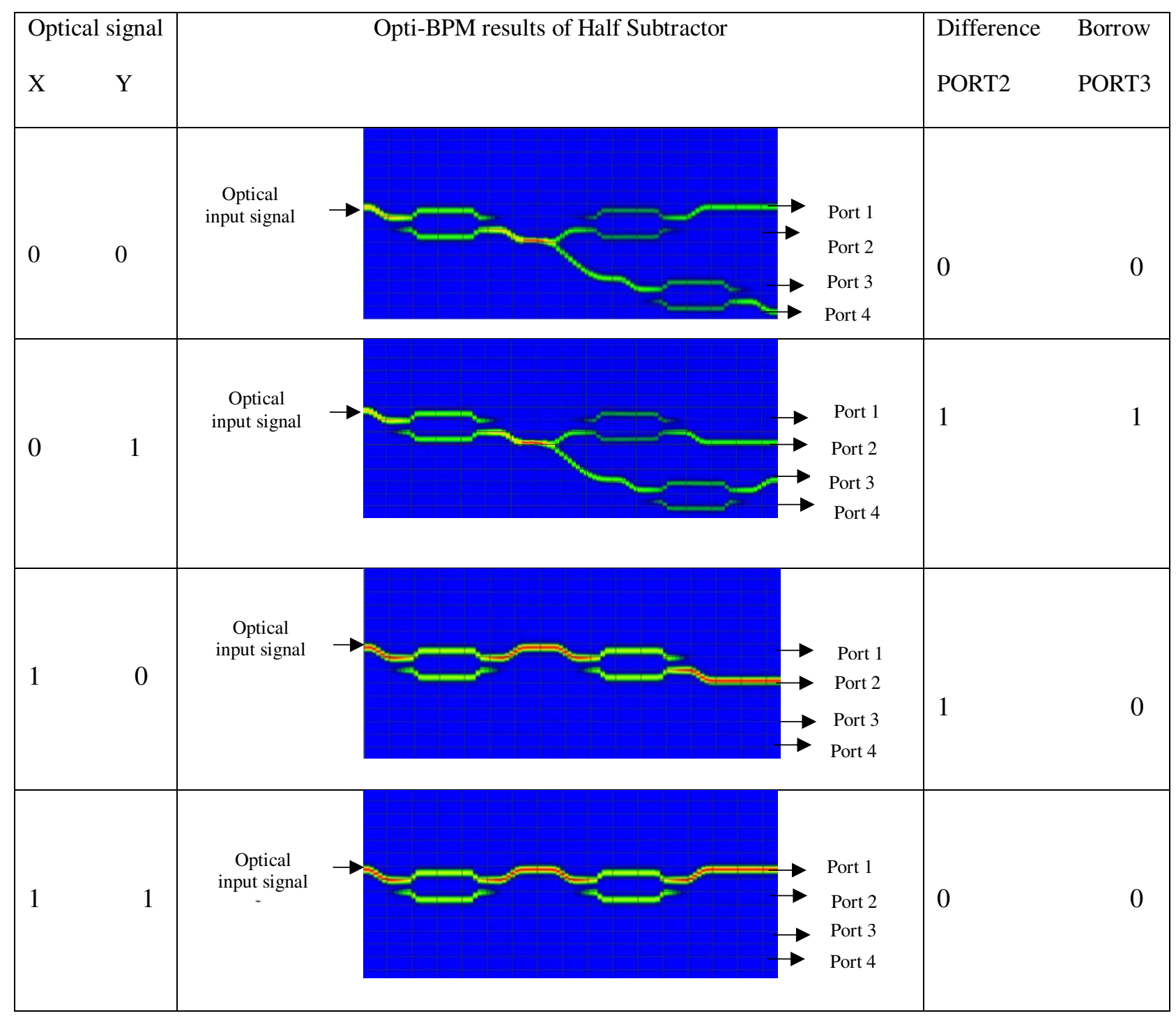



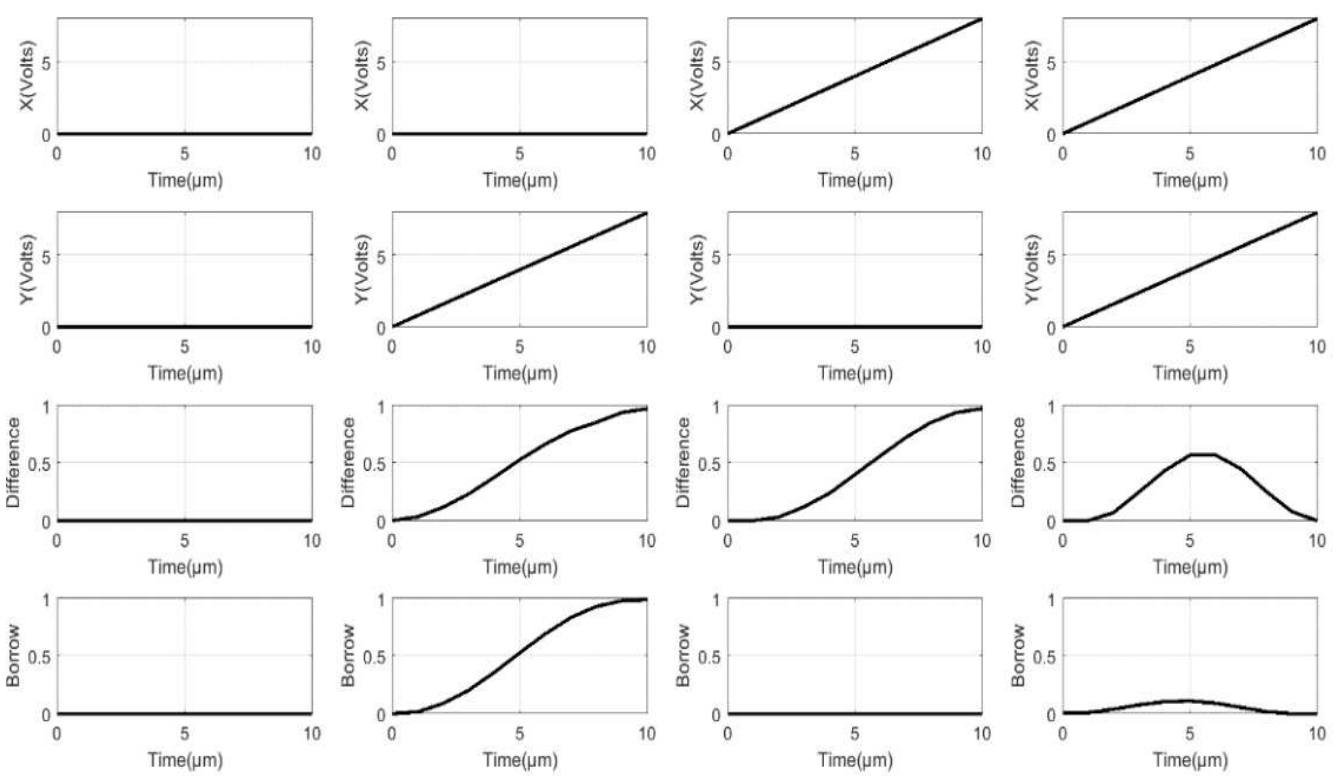

Fig. 5. MATLAB Simulation results for different combination control signals given to half-subtractor.

\section{Conclusion}

In conclusion, the proposed design discuss optical logic operation module such as half adder and half subtractor to perform optical signal processing. The paper includes a detailed discussion of half adder and half subtractor using electro-optic effect based mach-zehnder interferometer. The performance of the proposed device is carried out by analyzing the suitable mathematical expression and simulation results obtained from Opti-BPM and MATLAB software. Finally, the designed device provides a platform for all-optical signal processing, which is useful for different applications such as arithmetic logic units, encryption/decryption of secure network, binary counters and packet switching network.

\section{References}

1. W, Stefan., J. E, Benjamin.: All-Optical Signal Processing: Data communication and storage applications, Switzerland (2015)

2. C, Nelson., C, Addlfd.: Advances in laser and electro-optics, Intech (2010).

3. I, Nelson.: Semiconductor Integrated Optics for Switching light, USA (2017).

4. Tarek S. El-Bawab.: Optical switching, Springer Science, USA(2006).

5. Kumar, A., Kumar, S., Raghuwanshi, S.K.: Implementation of XOR/XNOR and AND logic gates by using Mach-Zehnder interferometers. Optik. 125, 5764-5767 (2014). https://doi.org/10.1016/j.ijleo.2014.07.037

6. Raghuwanshi, S.K., Kumar, A., Rahman, A.: Implementation of high speed optical universal logic gates using the electro-optic effect-based Mach-Zehnder interferometer structures. Journal of Modern Optics. 62, 978-988 (2015). https://doi.org/10.1080/09500340.2015.1015636

7. Sutanu Kumar Chandra, S.K.C.: All optical single module phase encoded half-adder and halfsubtractor exploiting four wave mixing in semiconductor optical amplifier. IOSR Journal of Electronics and Communication Engineering. 6, 67-71 (2013). https://doi.org/10.9790/2834-0626771 
8. Nady, M., Hussein, K.F.A., Ammar, A.E. hadi A.: Ultrafast all-optical full adder using quantum-dot semiconductor optical amplifier-based Mach-Zehnder interferometer. Progress In Electromagnetics Research B. 69-88 (2013). https://doi.org/10.2528/pierb13063006

9. Chakraborty, B., Mukhopadhyay, S.: All-optical method of developing half and full subtractors by the use of phase encoding principle. Optik. 122, 2207-2210 (2011). https://doi.org/10.1016/j.ijleo.2011.01.014

10. Kim, J.H., Byun, Y.T., Jhon, Y.M., Lee, S., Woo, D.H., Kim, S.H.: All-optical half adder using semiconductor optical amplifier based devices. Optics Communications. 218, 345-349 (2003). https://doi.org/10.1016/S0030-4018(03)01203-3

11. Neisy, M., Soroosh, M., Ansari-Asl, K.: All optical half adder based on photonic crystal resonant cavities. Photonic Network Communications. 35, 245-250 (2018). https://doi.org/10.1007/s11107-017-0736-6

12. Moradi, R.: All optical half subtractor using photonic crystal based nonlinear ring resonators. Optical and Quantum Electronics. 51, 1-9 (2019).

https://doi.org/10.1007/s11082-019-1831-y

13. McGeehan, J.E., Kumar, S., Willner, A.E.: Simultaneous optical digital halfsubtraction and -addition using SOAs and a PPLN waveguide. Optics Express. 15, 5543 (2007). https://doi.org/10.1364/oe.15.005543

14. Kumar, S., Willner, A.E., Gurkan, D., Parameswaran, K.R., Fejer, M.M.: All-optical half adder using an SOA and a PPLN waveguide for signal processing in optical networks. Optics Express. 14, 10255 (2006). https://doi.org/10.1364/oe.14.010255

15. Thongmee, S., Yupapin, P.P.: All optical half adder/subtractor using dark-bright soliton conversion control. Procedia Engineering. 8, 217-222 (2011). https://doi.org/10.1016/j.proeng.2011.03.040

16. Chattopadhyay, T.: Terahertz optical asymmetric demultiplexer (TOAD) based halfadder and using it to design all-optical flip-flop. Optik. 123, 1961-1964 (2012). https://doi.org/10.1016/j.ijleo.2011.11.012

17. MANDAL, P.: Method of Developing All Optical Half-Adder Based on Nonlinear Directional Coupler. Optics and Photonics Letters. 6, 1350001 (2013). https://doi.org/10.1142/s1793528813500019

18. Singh, P., Singh, A.K., Arun, V., Dixit, H.K.: Design and analysis of all-optical halfadder, half-subtractor and 4-bit decoder based on SOA-MZI configuration. Optical and Quantum Electronics. 48, 1-14 (2016). https://doi.org/10.1007/s11082-016-0429-x

19. Singh, S., Kaler, R.S., Kaur, R.: Realization of high speed all-optical half adder and half subtractor using SOA based logic gates. Journal of the Optical Society of Korea. 18, 639-645 (2014). https://doi.org/10.3807/JOSK.2014.18.6.639

20. Kumar, S., Singh, L., Raghuwanshi, S.K.: Design of plasmonic half-adder and halfsubtractor circuits employing nonlinear effect in Mach-Zehnder interferometer.

Journal of Computational Electronics. 16, 139-147 (2017). https://doi.org/10.1007/s10825-016-0927-x

21. Dai, B., Shimizu, S., Wang, X., Wada, N.: Simultaneous all-optical half-adder and half-subtracter based on two semiconductor optical amplifiers. IEEE Photonics Technology Letters. 25, 91-93 (2013). https://doi.org/10.1109/LPT.2012.2228847

22. Kaur, S., Kaler, R.S.: All optical integrated full adder-subtractor and demultiplexer using SOA-based Mach - Zehnder interferometer. International Journal of Engineering Science and Technology. 4, 303-310 (2012)

23. Gayen, D.K., Chattopadhyay, T.: Designing of optimized all-optical half adder circuit using single quantum-dot semiconductor optical amplifier assisted Mach-Zehnder 
interferometer. Journal of Lightwave Technology. 31, 2029-2035 (2013).

https://doi.org/10.1109/JLT.2013.2263251

24. Singh, K., Kaur, G.: All-Optical Half-Adder and Half-Subtracter Based on Semiconductor Optical Amplifier. Open Journal of Communications and Software. 2014, 42-51 (2014). https://doi.org/10.15764/cs.2014.01004

25. Kaur, S.: All-optical binary full subtractor using logic operations based on nonlinear properties of semiconductor optical amplifier. Journal of Nonlinear Optical Physics and Materials. 25, 1-9 (2016). https://doi.org/10.1142/S021886351650003X

26. Singh, G., P. Yadav, R., Janyani, V.: Ti indiffused Lithium Niobate (Ti: LiNbO3) Mach-Zehnder interferometer all optical switches: A review. New Advanced Technologies. (2010). https://doi.org/10.5772/9422

27. Kaur, Sehajpal \& Singh, Mandeep \& Gill, Harpuneet \& Priyanka, Priyanka \& Singh, Maninder. (2018). Performance Evaluation of Ti:LiNbO 3 Mach-Zehnder Interferometer Switch at $1.3 \mu \mathrm{m}, 1.46 \mu \mathrm{m}$ and $1.55 \mu \mathrm{m} .2233-2238$. 10.1109/ICRIEECE44171.2018.9008441.

28. OptiBPM 9.0 Technical Background and Tutorials, Waveguide Optics Modeling Software Systems, Optiwave, 2007. 
Figures

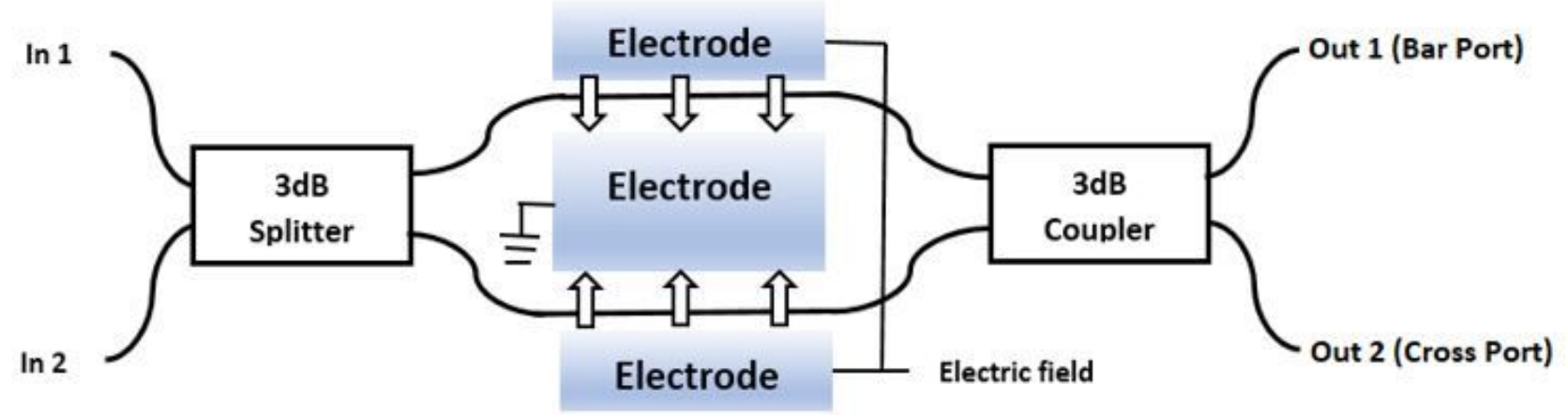

Figure 1

Schematic view of electro-optic MZI switch

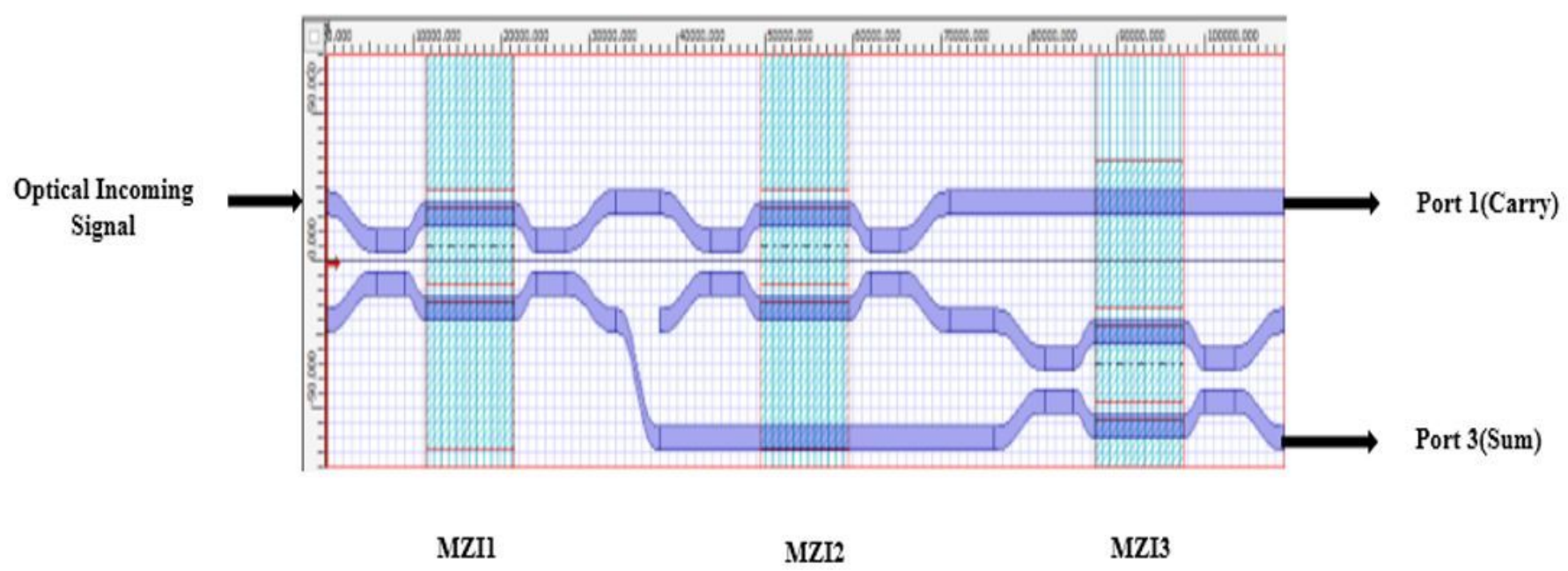

Figure 2

Opti-BPM layout of Half Adder using MZI switch 

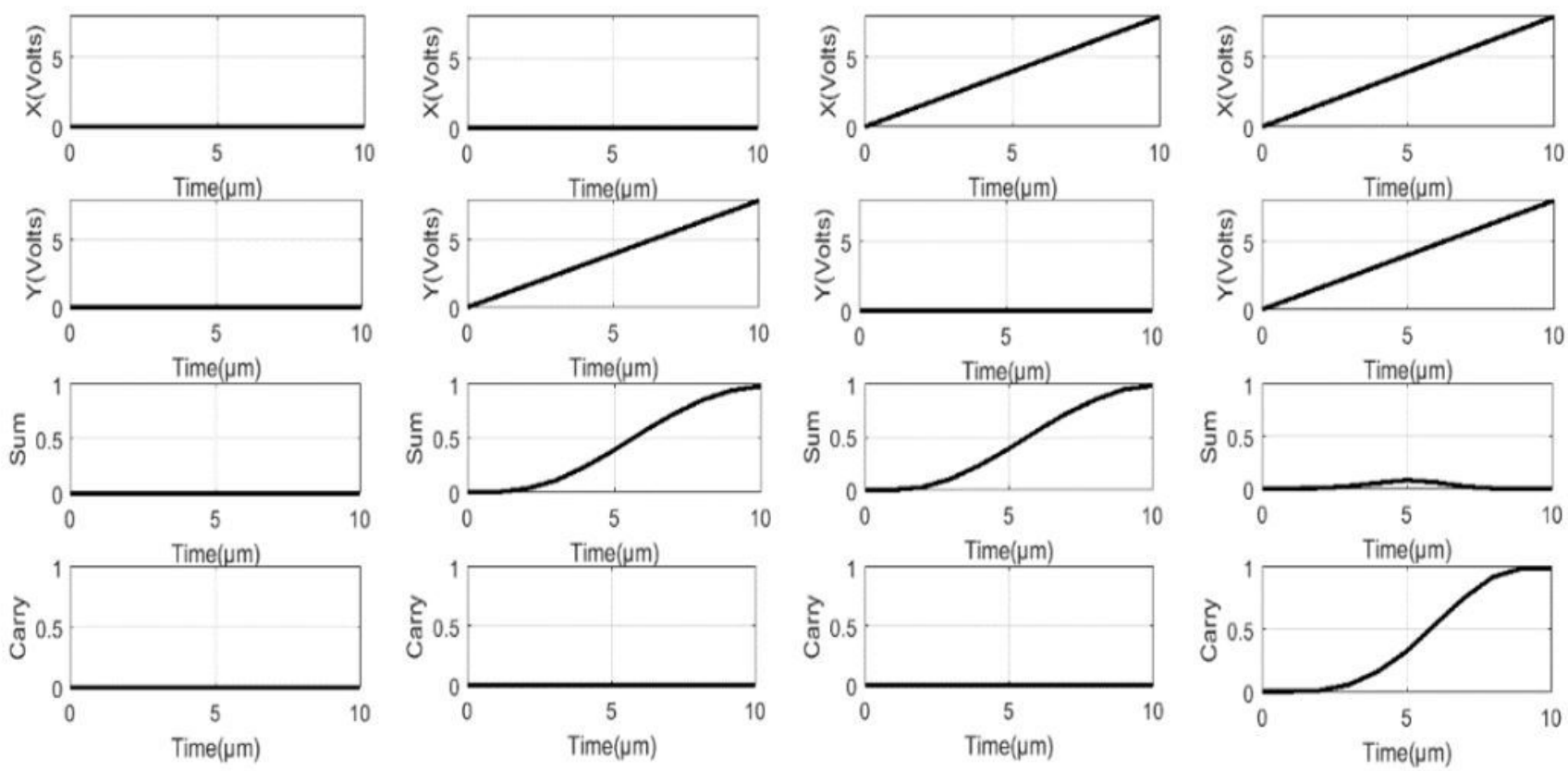

Figure 3

MATLAB simulation results of Half Adder.

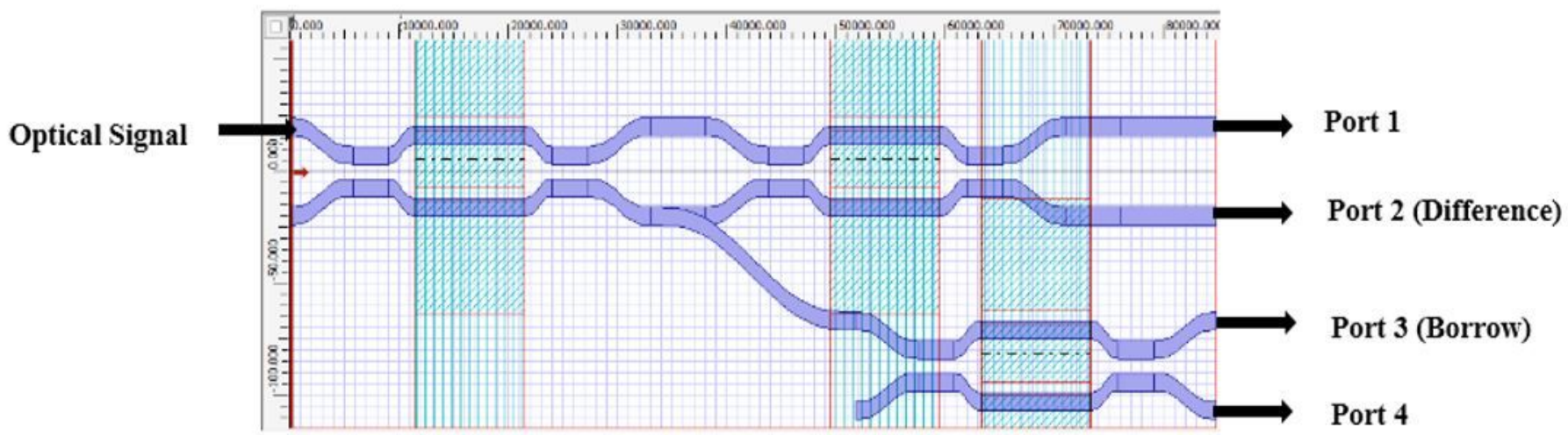

MZI 1

MZI 2 MZI 3

\section{Figure 4}

Layout of Half Subtractor 

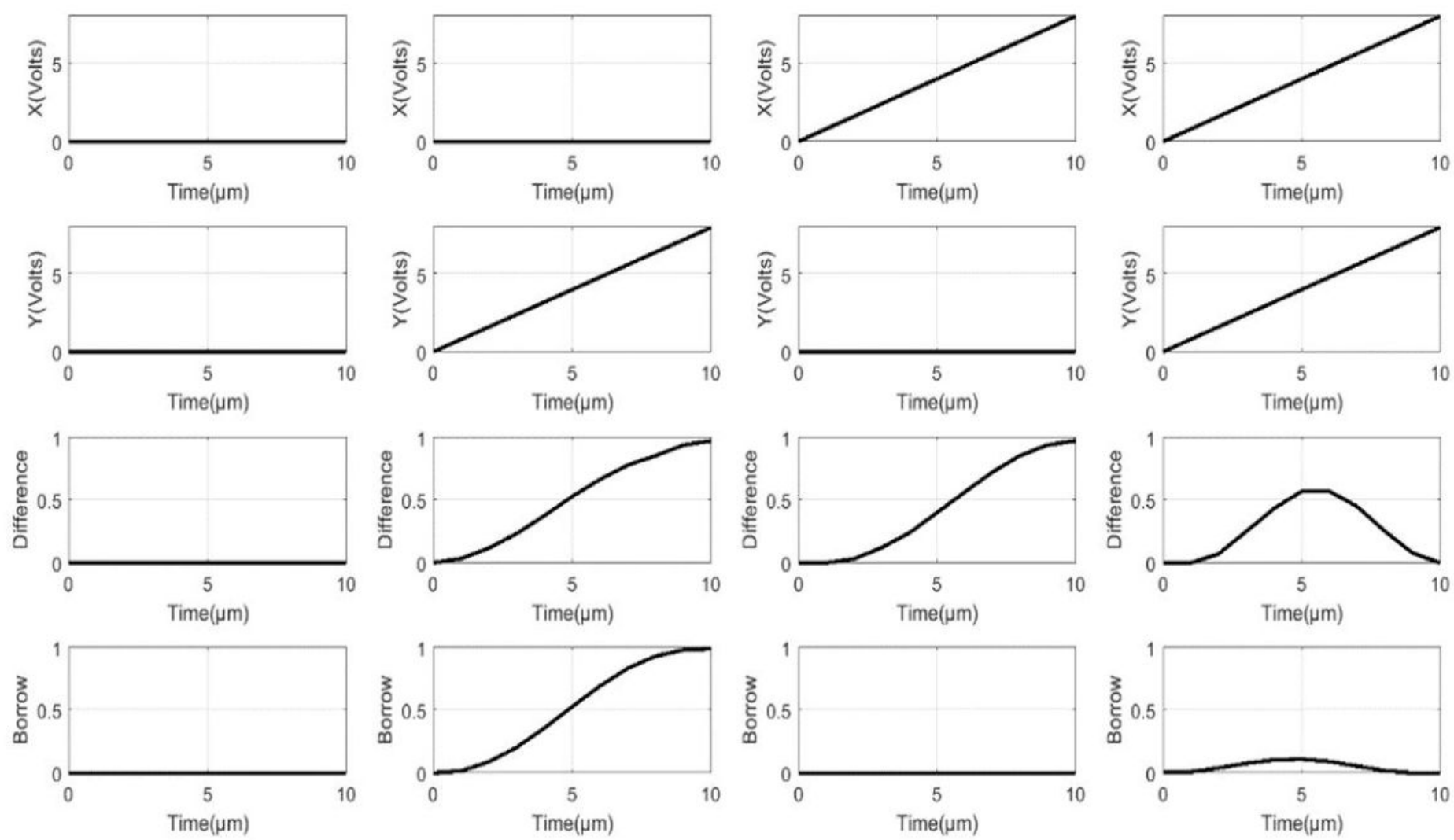

Figure 5

MATLAB Simulation results for different combination control signals given to half-subtractor. 